\title{
Isolated metastatic papillary thyroid carcinoma masquerading as parapharyngeal space paraganglioma
}

\author{
Suresh Chandra Sharma ${ }^{1}$, Ms, Prem Sagar ${ }^{1}$, MS, DNB, Rajeev Kumar ${ }^{1}$, MS, DNB, Prashant Durgapal², MD
}

\begin{abstract}
Isolated metastatic deposits of papillary thyroid carcinoma to the parapharyngeal space are rare. Herein, we describe the case of a young woman who presented with a right-sided oropharyngeal mass that was initially diagnosed as a parapharyngeal space paraganglioma. The patient opted for conservative treatment as she was asymptomatic and wished to avoid the risk of neurovascular morbidity associated with surgery. After 20 years, the patient sought treatment again for the oropharyngeal mass, which had progressively increased in size and was causing difficulty in swallowing. Repeat imaging of the affected area revealed that the mass had increased significantly in size; it also revealed the presence of a previously absent small lesion in the right lobe of the thyroid. Excision of the parapharyngeal space tumour and near-total thyroidectomy were performed; the excised specimens showed features of the follicular variant of papillary thyroid carcinoma. As papillary thyroid carcinoma that metastasises to the parapharyngeal space can masquerade as a paraganglioma, clinicians should bear in mind that an isolated metastatic deposit in the parapharyngeal space could be the first sign of occult papillary thyroid carcinoma.
\end{abstract}

Keywords: papillary thyroid carcinoma, paraganglioma, parapharyngeal space, parapharyngeal space tumour

\section{INTRODUCTION}

Isolated metastatic deposits of papillary thyroid carcinoma (PTC) to the parapharyngeal space (PPS) are rare, with very few cases reported in the literature. ${ }^{(1)}$ Herein, we describe the rare case of a 24-year-old woman who was misdiagnosed to have a paraganglioma, which was actually an isolated vascular metastatic deposit of an occult PTC to the PPS. The misdiagnosis was only corrected after 20 years. This report aims to highlight that isolated vascular metastatic deposits of PTC to the PPS can masquerade as a paraganglioma and may be the only presentation of an occult PTC.

\section{CASE REPORT}

A 24-year-old woman underwent tonsillectomy for tonsillar hypertrophy at a different institute in 1983. Intraoperatively, the surgeon noticed a bulge in her right tonsillar bed. The patient was informed of the finding and radiography was performed. Radiography revealed an enhancing mass lesion in the right PPS, which was suggestive of a paraganglioma. The thyroid gland was normal. Peroral fine needle aspiration cytology (FNAC) of the lesion revealed red blood cells, and groups and clumps of monomorphic-looking oval cells with regular nuclei. These cells were arranged in a small acinar fashion. Few foamy macrophages were also seen. No cytological atypia or anaplasia was seen in the epithelial cells. There was no evidence of a granulomatous lesion, and the features observed were suggestive of a benign epithelial tumour. The patient opted for conservative treatment instead of surgery in view of her asymptomatic status and the risk of postsurgery neurovascular morbidity.

However, 15 years later, the patient sought medical advice at our institute for the oropharyngeal mass, which had progressively increased in size. Contrast-enhanced computed tomography (CECT) confirmed that the vascular tumour in the right PPS had increased in size since the last performed imaging. There was no abnormality observed in her thyroid gland. The patient was once again offered surgery as a treatment option, but she opted to wait and observe. She had no signs or symptoms of lower cranial nerve palsy.

Five years later, the patient presented to our institute again for the progressive oropharyngeal mass, reporting difficulty in swallowing. On examination, a mucosa-covered mass that occluded almost three quarters of the oropharyngeal lumen was observed in the right tonsillar fossa. Based on what was documented in her clinical records, the oropharyngeal mass had significantly increased in size. There were no palpable neck nodes, and all lower cranial nerves were normal. Repeat CECT revealed a larger, vascular mass in the right PPS. In addition, there was a new finding of a small lesion in the right lobe of the thyroid (Fig. 1). There was no radiological evidence of metastatic cervical lymphadenopathy. The mass extended from the level of the pterygoid plates cranially and to the upper border

${ }^{1}$ Department of Otorhinolaryngology - Head and Neck Surgery, ${ }^{2}$ Department of Pathology, All India Institute of Medical Sciences, New Delhi, India

Correspondence: Dr Suresh Chandra Sharma, Professor and Head, Department of Otorhinolaryngology - Head and Neck Surgery, ENT Office, 4th Floor, Teaching Block, All India Institute of Medical Sciences, Ansari Nagar, New Delhi 110029, India. suresh6sharma@yahoo.com 

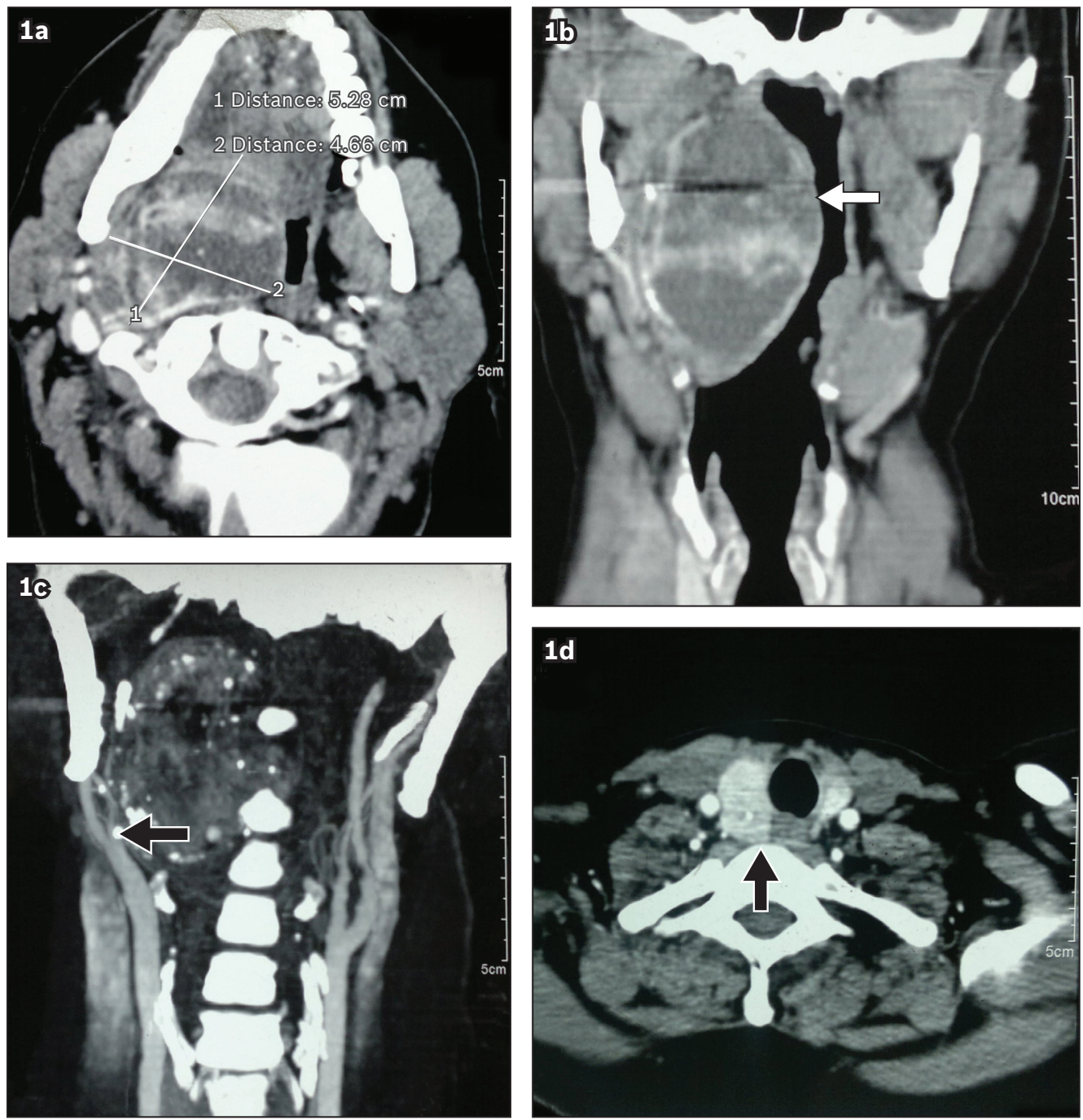

Fig. 1 (a) Axial contrast-enhanced computed tomography (CECT) of the neck shows an approximately $5 \mathrm{~cm} \times 4 \mathrm{~cm}$ enhancing mass in the right parapharyngeal space (PPS), with areas of internal calcification. The mass is seen pushing the carotid vessels anterolaterally and the oropharyngeal lumen is significantly narrowed. (b) Coronal CECT of the neck shows the large, enhancing mass (arrow) extending from the level of pterygoid plates cranially and to the thyroid cartilage caudally. (c) Posterior-view CT angiogram of the neck shows the displacement of the carotid vessels (arrow) by the mass. (d) Axial CECT of the neck shows a small nodule (arrow) in the right lobe of the thyroid gland.

of the thyroid cartilage caudally. It was heterogeneously enhancing, with internal areas of irregular calcifications. The great vessels were pushed anterolaterally by the tumour.

Based on the patient's long clinical history, presentation of the pharyngeal mucosal bulge and imaging findings of an enhancing vascular lesion with anterolateral displacement of the great vessels, a probable diagnosis of paraganglioma was made. However, as ultrasonography-guided FNAC of the thyroid lesion showed features suggestive of PTC, excision of the PPS tumour with near-total thyroidectomy was performed. To our surprise, histopathology revealed features of the follicular variant of PTC in both excised specimens (Fig. 2), confirming that the tumours were PTCs.

Postoperatively, the patient underwent whole-body radionuclide imaging with iodine-131, revealing bilateral multiple metastatic deposits to the lungs. Complete resolution of the lesions was achieved using radionuclide iodine-131.
Subsequent thyroid scans showed no uptake at the primary, regional or distant sites. The patient has remained diseasefree for the last nine years and is being screened annually for recurrence.

\section{DISCUSSION}

PPS tumours account for only $0.5 \%$ of all head and neck tumours. ${ }^{(2)}$ Tumours in the PPS arise from either the adjacent structures, the contents of the space, or rarely, metastatic deposits from distant primary sites. While minor salivary gland tumours are the most commonly reported tumour in the prestyloid compartment, poststyloid compartment tumours are mostly neurogenic in origin. ${ }^{(3)}$

The prestyloid compartment has been designated as the true PPS. ${ }^{(4)}$ The contents of this space include adipose tissue, minor salivary gland rests, arteries and veins. Primary lesions that arise in this space are mainly lipomas and minor salivary 

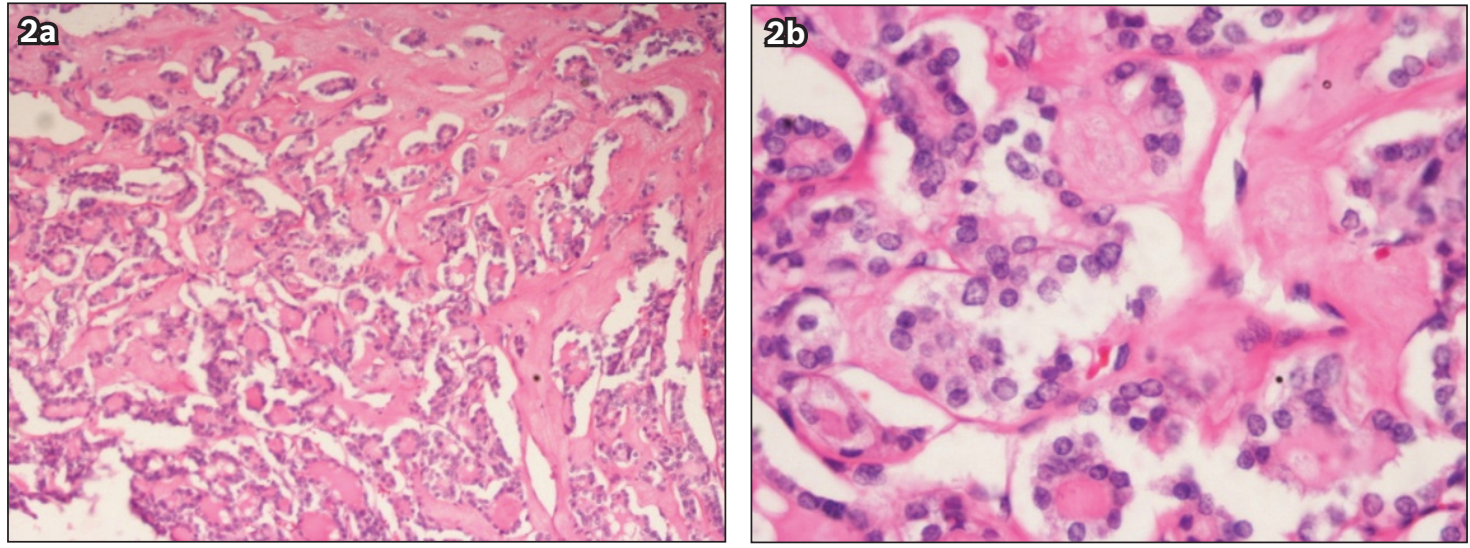

Fig. 2 Photomicrographs of the excised specimens show features of the follicular variant of papillary thyroid carcinoma. (a) Low magnification (Haematoxylin \& eosin, $\times 10$ ) and (b) medium magnification (Haematoxylin \& eosin, x 40).

gland tumours. These lesions are usually diagnosed based on characteristic features on radiography. Smaller lesions usually have fat lining their periphery, and no fat displacement. Larger lesions, including benign and malignant minor salivary gland tumours, are generally well defined and may sometimes cause fat displacement. To facilitate the preoperative diagnosis of malignant lesions, which might be difficult, tumour infiltrated fat is generally taken to indicate malignant nature. Although tumours arising in the deep lobe of the parotid gland (mainly pleomorphic adenoma) also lie in the prestyloid compartment, these cause the anteromedial displacement of PPS fat, and no fat plane exists between the tumour and the deep lobe of the parotid gland. Tumours of the prestyloid compartment lie anterior to the internal carotid artery. ${ }^{(4)}$

The poststyloid compartment has been designated as the carotid space. ${ }^{(4)}$ The commonly seen tumours in this space are paragangliomas and nerve sheath tumours (e.g. schwannomas and neurofibromas), and rarely, nodal metastatic deposits from distant sites. On radiology (CECT or magnetic resonance imaging), paragangliomas are highly vascular and enhance intensely, with diagnostic flow voids if the tumour is larger than $2 \mathrm{~cm}$ in size. Carotid body paragangliomas are located at the bifurcation of the common carotid artery and cause characteristic splaying of the external and the internal carotid arteries. In contrast, vagale paragangliomas and glomus jugulare tumours cause the anterior displacement of PPS fat and the internal carotid artery, and the lateral displacement of the internal jugular vein. Nerve sheath tumours lack flow voids even when larger in size, and may or may not enhance with contrast. Schwannomas are generally heterogenous owing to cystic changes or haemorrhage. ${ }^{(4)}$

PTC usually presents as a palpable neck mass arising from the thyroid or as metastatic regional lymphadenopathy. ${ }^{(3)}$ Other rare presentations in the presence of a clinically normal thyroid gland include hoarseness, vocal cord palsy, haemoptysis and dyspnoea. ${ }^{(3)}$ PTC usually metastasises to the lungs and bones. ${ }^{(1)}$ It very rarely presents as a PPS mass in the neck or an oropharyngeal mass. ${ }^{(3)}$ In a case series by Qui et al, the authors reported an incidence of $2.5 \%$ for metastatic deposits of differentiated thyroid carcinomas in the PPS detected using iodine-131 single-photon emission computed tomography/computed tomography. ${ }^{(1)}$ Metastatic deposits to the PPS can arise from primary tumours located in the breast, lung, kidney, bone, colon, prostate and thyroid. ${ }^{(5)}$ However, the presence of internal calcification in metastatic deposits narrows the primary site to the breast, bone, prostate or papillary thyroid carcinoma. ${ }^{(6,7)}$ In the present case, the occult thyroid primary tumour led to the erroneous initial clinical diagnosis of PPS paraganglioma.

The pathophysiology of metastatic deposits of PTC to the PPS remains controversial. The existence of an ectopic parapharyngeal thyroid gland in the presence of a normally located, functioning thyroid gland has been reported in the literature. ${ }^{(8)}$ In such cases, malignant transformation of the ectopic thyroid tissue could be a possibility. Direct extension of the primary PTC from the superior pole to the lower part of the PPS through a narrow stalk along the neurovascular bundle has also been reported. ${ }^{(3)}$ Another possible route of dissemination is explained by Rouviere's anatomical pathway. In this pathway, a lymphatic channel connects the posterior and superior thyroid lymph vessels to the retropharyngeal lymphatic system. ${ }^{(9)}$ The sinus of Morgagni allows the parapharyngeal and retropharyngeal spaces to communicate with each other. ${ }^{(10)}$

PPS tumours are usually primary in origin, and only rarely does the PPS serve as a nest for distant metastasis. Generally, the tissue of origin of PPS tumours is diagnosed radiographically, in conjunction with the patient's clinical signs and symptoms. FNAC is rarely performed for vascular tumours, and at times, it may be inconclusive. Thus, in the absence of any signs or symptoms from the primary tumour, a high index of suspicion is required to consider a metastatic origin for the PPS lesion. Surgery is the treatment of choice for PPS tumours, as was done in the present case.

In conclusion, while PPS tumours are rare tumours of the head and neck region, metastatic deposits from distant primary sites are even rarer. Metastatic deposits from an occult PTC presenting as an oropharyngeal mass has rarely been described as the initial presentation for PTC. However, as observed in our patient, an isolated vascular metastatic deposit from an occult 
PTC can masquerade as a paraganglioma and thus affect treatment strategy. Clinicians should bear this in mind when attending to patients presenting with lesions in the PPS.

\section{REFERENCES}

1. Qiu ZL, Xu YH, Song HJ, Luo QY. Localization and identification of parapharyngeal metastases from differentiated thyroid carcinoma by 131I-SPECT/CT. Head Neck 2011; 33:171-7.

2. Stell PM, Mansfield AO, Stoney PJ. Surgical approaches to tumors of the parapharyngeal space. Am J Otolaryngol 1985; 6:92-7.

3. Cetik F, Yazici D, Uguz A. A thyroid tumor extending to the parapharyngeal space. BMC Ear Nose Throat Disord 2006; 6:3.

4. Stambuk HE, Patel SG. Imaging of the parapharyngeal space. Otolaryngol
Clin North Am 2008; 41:77-101.

5. Hirshberg A, Buchner A. Metastatic tumours to the oral region. An overview. Eur J Cancer B Oral Oncol 1995; 31:355-60.

6. Som PM. Detection of metastasis in cervical lymph nodes: CT and MR criteria and differential diagnosis. AJR Am J Roentgenol 1992; 158:961-9. 7. Langlais $R$, Langland $O$, Nortje $C$, eds. Diagnostic imaging of the jaws. Baltimore: Williams and Wilkins, 1995, p406-10.

8. Feisal TK, Prepageran N, Shahrizal T, Zulkiflee AB. Unusual parapharyngeal lesion: aberrant thyroid gland. Singapore Med J 2008; 49:e137-8.

9. Thomas G, Pandey M, Jayasree K, et al. Parapharyngeal metastasis from papillary microcarcinoma of thyroid: report a case diagnosed by peroral fine needle aspiration. Br J Oral Maxillofac Surg 2002; 40:229-31.

10. Saydam L, Kalcioglu T, Demirkiran A, Gurer M. Occult papillary thyroid carcinoma presenting as a parapharyngeal metastasis. Am J Otolaryngol 1999; 20:166-8. 